Copyright 2001 American Association of Physics Teachers (AIP Publishing). This article may be downloaded for personal use only. Any other use requires prior permission of the author and AIP Publishing.

The following article appeared as: Penner, A.R. (2001). The physics of sliding cylinders and curling rocks. American Journal of Physics, 69(3), 332-339, and may be found at http://dx.doi.org/10.1119/1.1309519 


\title{
The physics of sliding cylinders and curling rocks
}

\author{
A. Raymond Penner \\ Physics Department, Malaspina University-College, Nanaimo, British Columbia V9R 5S5, Canada
}

(Received 2 February 2000; accepted 7 June 2000)

\begin{abstract}
The lateral deflection of a rotating cylindrical shell sliding on one of its ends is considered and both theoretical and experimental results are presented. The coefficient of kinetic friction between a curling rock and an ice surface is then derived and compared with experiment. Current models of the motion of a curling rock are discussed and an alternate hypothesis is presented. (C) 2001 American Association of Physics Teachers.
\end{abstract}

[DOI: 10.1119/1.1309519]

\section{INTRODUCTION}

The motion of a cylindrical shell or hollow cylinder sliding on one of its ends along a dry horizontal surface is an elementary problem in physics. Kinetic friction acts to oppose the motion and the cylinder decelerates and comes to rest. An additional feature arises, however, if the cylinder is given an initial angular velocity about its axis. The cylinder is then deflected laterally from its original direction of motion. This behavior, as far as I know, has not been formally analyzed, although Daniels ${ }^{1}$ has made reference to it. This paper will derive the equations of motion for such a rotating cylinder sliding on its end and compare the resulting theoretical path with experiment.

A practical example of such motion may be found in the game of curling. The game involves propelling an $18-\mathrm{kg}$ disk-shaped granite rock down a sheet of ice with the correct velocity so that the rock comes to rest approximately $28 \mathrm{~m}$ away in a set of painted rings. As the curling rock's running surface is a band or annulus, referred to in curling as its running band, it would be expected to behave similarly to the above-mentioned cylindrical shell. The curling rock is given an initial rotational velocity during the throwing motion which does indeed cause the rock to deflect sideways, or curl, from its original direction. The overall deflection is on the order of $1 \mathrm{~m}$ and is a key part of the game as one tries to curl the rock around previously played ones in order to protect them from the opposition's rocks. There is, however, a fundamental difference between the curling rock and the rotating cylinder. A rotating cylinder sliding on a dry surface curls in the opposite sense to the rotation about its axis while a curling rock sliding on ice curls in the same sense as its body rotation.

This behavior of a curling rock has previously been considered and two general theories for the curling of a rock have been presented. Johnston ${ }^{2}$ and Shegelski, Niebergall, and Watton ${ }^{3}$ have explained the curl as being due to a frontback asymmetry in the frictional force between a curling rock and the ice. Denny ${ }^{4}$ has presented a model based on the curl being due to a left-right asymmetry in the frictional force. Unfortunately, no experimental results were given to support these models. This paper will look at the curling phenomena by first providing a theoretical model for the kinetic friction between a curling rock and ice which will be shown to be consistent with experimental results. The various curling models will then be discussed and compared with experiment. Finally, observations and an alternate hypothesis will be presented.

\section{EQUATIONS OF MOTION}

Figure 1 shows the geometry that will be used for a cylindrical shell of height $h$, average radius $R$, and running bandwidth (i.e., wall thickness) $b$ sliding on one of its ends across a flat surface. The $y$ axis is taken to be along the initial direction of motion, while the $x$ axis is in the lateral direction, and the $z$ axis is perpendicular to the sliding surface. The initial starting position for the center of mass is taken to be $x=y=0$, the initial translational velocity components are $v_{x}=0$ and $v_{y}=v_{y 0}$, and the initial angular velocity $\omega$ $=\omega_{0}$.

The velocity $\mathbf{u}$ at an angular position $\theta$ along the sliding end or running band of the cylinder will be given by

$$
\begin{aligned}
\mathbf{u} & =\left(v_{x}-\omega R \sin \theta\right) \mathbf{i}+\left(v_{y}+\omega R \cos \theta\right) \mathbf{j} \\
& =u_{x} \mathbf{i}+u_{y} \mathbf{j} .
\end{aligned}
$$

The velocity at several positions around the running band is shown in Fig. 1(b). The frictional force per unit area, $\mathbf{f}(\theta)$, at a given position $\theta$ along the running band is then given by

$$
\begin{aligned}
\mathbf{f}(\theta) & =-\mu_{k} F_{n}(\theta) \mathbf{u}_{\mathbf{1}} \\
& =-\mu_{k} F_{n}(\theta)\left(u_{1 x} \mathbf{i}+u_{1 y} \mathbf{j}\right),
\end{aligned}
$$

where $\mu_{k}$ is the coefficient of kinetic friction, $F_{n}(\theta)$ is the normal force per unit area, and $\mathbf{u}_{\mathbf{1}}$ is the unit vector in the direction of the velocity at the given position.

For a cylinder of mass $m$ and moment of inertia $I$ about the central axis the resulting equations of motion will be

$$
\begin{aligned}
& \Sigma F=\int\left(F_{n}(\theta) \mathbf{k}+\mathbf{f}(\theta)\right) R b d \theta-m g \mathbf{k}=m a_{x} \mathbf{i}+m a_{y} \mathbf{j}, \\
& \Sigma \tau=\int \mathbf{r} \times\left(F_{n}(\theta) \mathbf{k}+\mathbf{f}(\theta)\right) R b d \theta=I \alpha \mathbf{k},
\end{aligned}
$$

where $\mathbf{r}$ is the position vector from the center of mass of the cylinder to the sliding surface. For a vertical cylinder of radius $R$ and height $h$,

$$
\begin{aligned}
\mathbf{r} & =R \cos \theta \mathbf{i}+R \sin \theta \mathbf{j}-h / 2 \mathbf{k} \\
& =r_{x} \mathbf{i}+r_{y} \mathbf{j}+r_{z} \mathbf{k} .
\end{aligned}
$$

Substituting Eqs. (2b) and (4b) into Eqs. (3a) and (3b) leads to the following six coupled equations:

$$
\int F_{n}(\theta) R b d \theta=m g,
$$




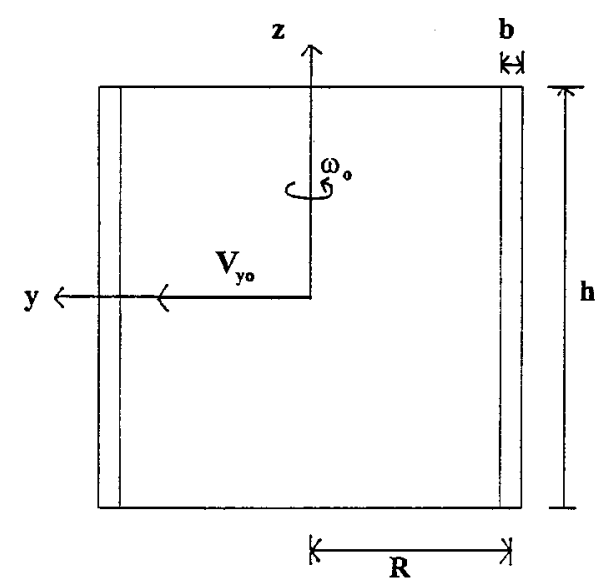

(a)

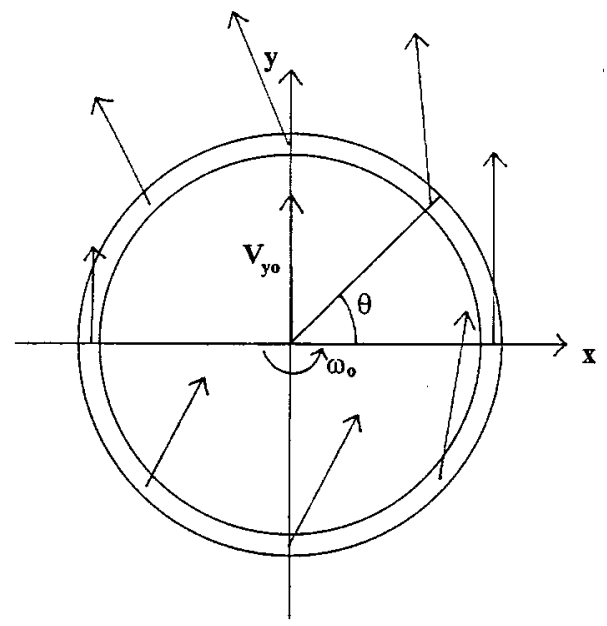

(b)

Fig. 1. (a) A cylindrical shell of radius $R$, height $h$, and running bandwidth $b$ with initial translational velocity $v_{y 0}$, and angular velocity $\omega_{0}$. The velocity at various points around the running band is shown in (b).

$$
\begin{aligned}
& -\int \mu_{k} F_{n}(\theta) u_{1 x} R b d \theta=m a_{x}, \\
& -\int \mu_{k} F_{n}(\theta) u_{1 y} R b d \theta=m a_{y}, \\
& \int F_{n}(\theta)\left(r_{x}+\mu_{k} u_{1 x} r_{z}\right) R b d \theta=0, \\
& \int F_{n}(\theta)\left(r_{y}+\mu_{k} u_{1 y} r_{z}\right) R b d \theta=0, \\
& -\int F_{n}(\theta)\left(\mu_{k} r_{x} u_{1 y}-\mu_{k} r_{y} u_{1 x}\right) R b d \theta=I \alpha .
\end{aligned}
$$

In order to solve the above equations and determine the resulting path of the cylinder the following functional form for the normal force, $F_{n}(\theta)$, will be assumed:

$$
F_{n}(\theta)=\beta+\gamma \sin \theta .
$$

Although other expressions for $F_{n}(\theta)$ could be used, the sinusoidal dependence was found to give a good description of the observed behavior and is consistent with the expectation that the normal force is greater at the front edge of the cylinder than at the back. Substituting Eq. (6) into Eqs. (5a) and $(5 \mathrm{~d})$ leads to

$\beta=m g / 2 \pi R b$,

$$
\gamma=-\int \beta\left(r_{x}+\mu_{k} u_{1 x} r_{z}\right) d \theta / \int \sin \theta\left(r_{x}+\mu_{k} u_{1 x} r_{z}\right) d \theta .
$$

Then, the accelerations of the cylinder are found from Eqs. (5b), (5c), and (5f):

$$
\begin{aligned}
& a_{x}=-\int \mu_{k}(\beta+\gamma \sin \theta) u_{1 x} R b d \theta / m, \\
& a_{y}=-\int \mu_{k}(\beta+\gamma \sin \theta) u_{1 y} R b d \theta / m, \\
& \alpha=-\int(\beta+\gamma \sin \theta)\left(\mu_{k} r_{x} u_{1 y}-\mu_{k} r_{y} u_{1 x}\right) R b d \theta / I .
\end{aligned}
$$

For a given coefficient of kinetic friction Eqs. (7b) and (8a)(8c) can be solved by numeric integration. The velocity and position of the cylinder as it slides along the surface are then determined by

$$
\begin{aligned}
& v_{x}(t+\Delta t)=v_{x}(t)+a_{x} \Delta t, \\
& v_{y}(t+\Delta t)=v_{y}(t)+a_{y} \Delta t, \\
& \omega(t+\Delta t)=\omega(t)+\alpha \Delta t, \\
& x(t+\Delta t)=x(t)+0.5\left(v_{x}(t)+v_{x}(t+\Delta t)\right) \Delta t, \\
& y(t+\Delta t)=y(t)+0.5\left(v_{y}(t)+v_{y}(t+\Delta t)\right) \Delta t .
\end{aligned}
$$

The incremental value, $\Delta t$, used for the theoretical paths shown in this paper was set at $0.001 \mathrm{~s}$, which results in an uncertainty in the lateral deflections of the cylinders of approximately $0.1 \%$.

\section{SLIDING CYLINDER}

\section{A. Theoretical path}

In the case of a cylindrical shell sliding on end along a dry solid surface it will be assumed that $\mu_{k}$, the coefficient of kinetic friction, is constant. Although $\mu_{k}$ may be uniform around the running band, the torque about the center of mass due to the frictional force will cause the normal force to be greater at the front of the cylinder than at the back, as shown in Fig. 2. Therefore, the magnitude of the frictional force, $f(\theta)$, will not be uniform around the running band. As an example, consider the motion of a vertical cylindrical shell of mass $250 \mathrm{~g}$, average radius $5 \mathrm{~cm}$, and a height of $10 \mathrm{~cm}$. For an initial velocity of $2 \mathrm{~m} / \mathrm{s}$, an initial angular velocity of $10 \mathrm{rad} / \mathrm{s}$, and with a constant coefficient of kinetic friction equal to 0.2 , it is found using the above equations that the normal force at the front of the cylinder is on average approximately two times greater than at the back of the cylinder. Therefore, the resulting frictional force will be greater at the front of the running band and, if the cylinder is rotating counterclockwise, the net result will be a lateral force to the right as the cylinder slides along the table, as shown in Fig. 


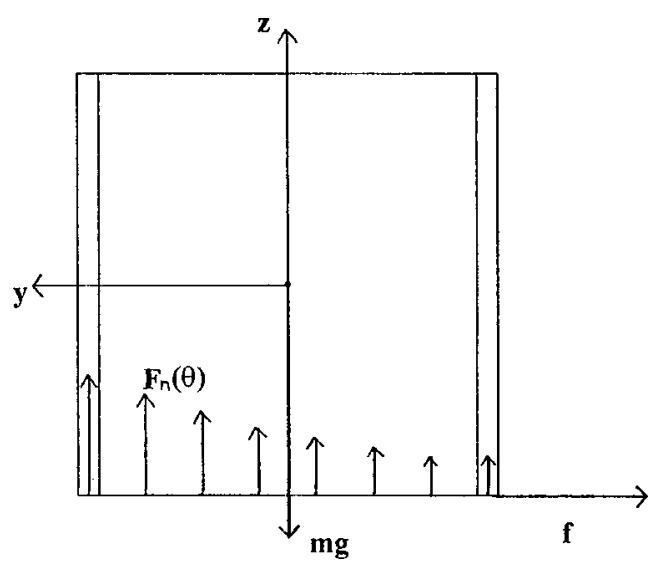

Fig. 2. The forces which act on the cylindrical shell including the variation of the normal force around the running band.

3. Figure 4 shows the calculated path using Eqs. (7)-(9) for the given cylinder along with similar cylinders having initial angular velocities of 2 and $20 \mathrm{rad} / \mathrm{s}$. The lateral displacement is found to be approximately proportional to the initial angular velocity. Figure 5 shows the calculated path for cylinders of various heights but with the same initial translational and rotational velocities. As expected the normal force at the front of the band will increase with the vertical position of the center of mass, which will lead to a greater frictional difference between the front and the back resulting in a greater lateral deflection.

\section{B. Experimental results}

In order to test the above theoretical model the actual paths of sliding cylinders were determined. This was done by lightly sprinkling corn starch on a bench and sliding a vertical pipe section down the bench with various initial translational and angular velocities. The corn starch did not have a noticeable effect on the motion of the cylinder but allowed the trail of the rotating cylinder to be visible so that its lateral deflection from a straight path could be determined. The main uncertainty in the measurement of the deflection was the determination of the tangent to the path at the starting point. An estimate of this uncertainty was obtained by varying the tangent within a reasonable range. The uncertainty shown with the experimental data is derived from this esti-

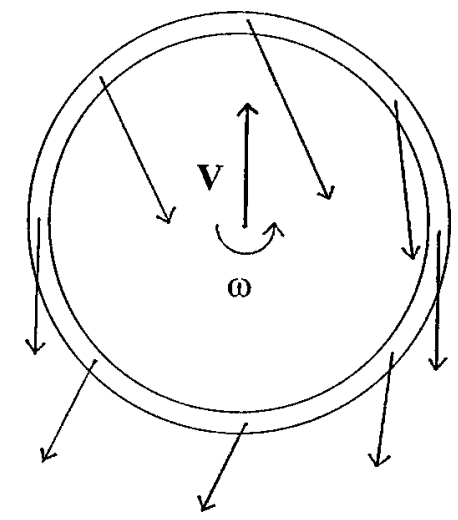

Fig. 3. The resulting frictional force at various points around the running band for a rotating cylindrical shell with constant $\mu_{k}$ and increased normal force on the leading half of the band.

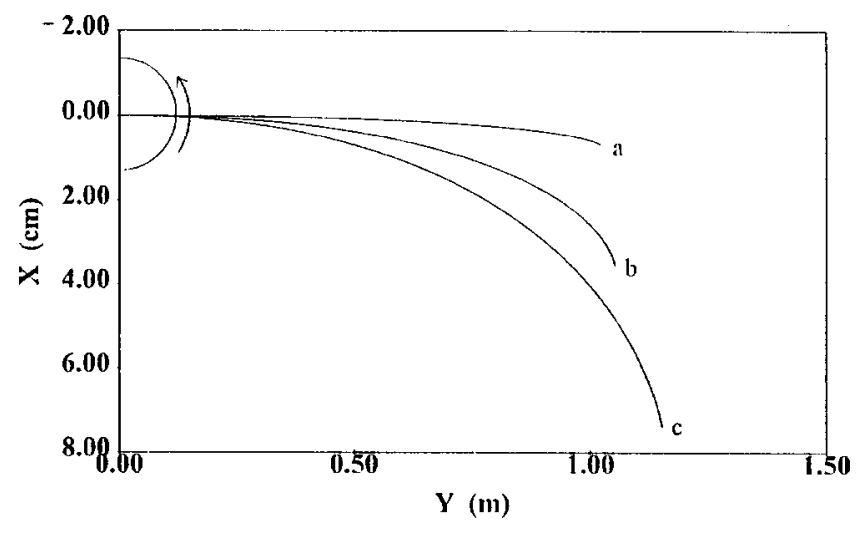

Fig. 4. The theoretical path of a cylindrical shell with $v_{y 0}=2 \mathrm{~m} / \mathrm{s}$ and with $\omega_{0}=2 \mathrm{rad} / \mathrm{s}$ (a), $10 \mathrm{rad} / \mathrm{s}(\mathrm{b})$, and $20 \mathrm{rad} / \mathrm{s}(\mathrm{c})$.

mate. The initial translational and angular velocities of the cylinders were obtained by mounting a video camera over the starting point and using a computer software package which allowed one to mark the position of each of the cylinders every $1 / 30 \mathrm{~s}$. The trials went well as long as one did not try to make the angular velocity too large, in which case the cylinder ended up with a wobble. A plastic pipe section was used which had a mass of $234 \mathrm{~g}$, a height of $9.17 \mathrm{~cm}$, a wall width of $0.63 \mathrm{~cm}$, and an average radius of $4.77 \mathrm{~cm}$. The pipe, however, was actually sliding on a narrow band near the outside edge of radius $5.00 \mathrm{~cm}$ and this is the value used for $R$. The coefficient of kinetic friction was determined from the sliding distances of the trials. A value of 0.235 \pm 0.005 was obtained which compares well with the value of $0.22 \pm 0.01$ obtained using a mass-pulley system to determine the force required to pull the cylinder along the table at constant velocity. Figure 6 shows the paths of two of the trials along with the theoretical paths. As is seen, there is good agreement and it can be concluded that the lateral deflection of a rotating sliding cylindrical shell is due to the difference between the normal force at the front and trailing sections of the running band.

\section{CURLING ROCK}

\section{A. Effect of the normal force}

Figure 7 shows the profile of a typical curling rock of mass $18 \mathrm{~kg}$, a height of $13 \mathrm{~cm}$, and a radius of $14.5 \mathrm{~cm}$. The

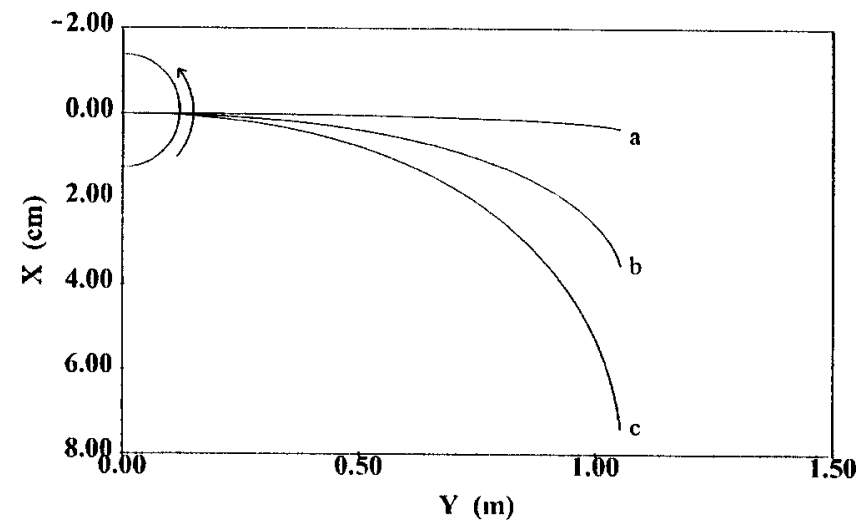

Fig. 5. The theoretical path of a cylindrical shell with $v_{y 0}=2 \mathrm{~m} / \mathrm{s}, \omega_{0}$

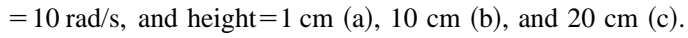




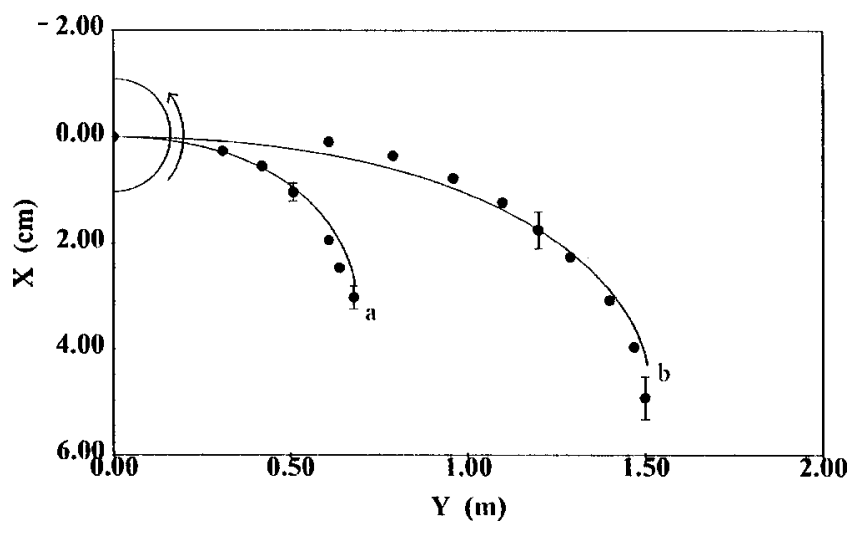

Fig. 6. The experimental positions of two sliding pipe sections as compared with the corresponding theoretical paths for $v_{y 0}=1.74 \mathrm{~m} / \mathrm{s}$ with $\omega_{0}$ $=10.2 \mathrm{rad} / \mathrm{s}$ (a) and for $v_{y 0}=2.61 \mathrm{~m} / \mathrm{s}$ with $\omega_{0}=10.6 \mathrm{rad} / \mathrm{s}(\mathrm{b})$.

running band has a radius of approximately $6.0 \mathrm{~cm}$ and a width of approximately $5.0 \mathrm{~mm}$. The same set of theoretical equations that was used for the cylindrical shell was applied to a rock with an initial velocity of $2.0 \mathrm{~m} / \mathrm{s}$ and angular velocity of $1.5 \mathrm{rad} / \mathrm{s}$, corresponding to values found in the game, and with a coefficient of kinetic friction of 0.0073 . The result is the rock traveling $28 \mathrm{~m}$ down the ice in $20 \mathrm{~s}$ and deflecting laterally approximately $8 \mathrm{~mm}$ in the same sense as the previously discussed cylinder. The normal force itself varies around the running band by less than $3 \%$. As a curling rock will actually curl approximately $1 \mathrm{~m}$ and in the opposite direction, a different explanation than the one given for the rotating cylinder sliding on a dry surface will be required to explain its deflection.

\section{B. Dependence on angular velocity}

In addition to curling in the opposite direction to that of the rotating cylinder, the deflection of a curling rock also has a different dependence on angular velocity. Various curling handbooks give instructions on how much initial angular velocity should be given to a curling rock. This ranges from having the rock completing one to two rotations ${ }^{5}$ while traveling the length of the ice to completing from two to four rotations. ${ }^{6}$ This corresponds to initial angular velocities ranging from approximately 0.3 to $1.5 \mathrm{rad} / \mathrm{s}$. It is also stated ${ }^{6}$ that if a rock has too great of a spin it will travel farther and it will curl less. Such a thrown rock is referred to as a spinner and is often the mistake of a novice. The reason such a rock

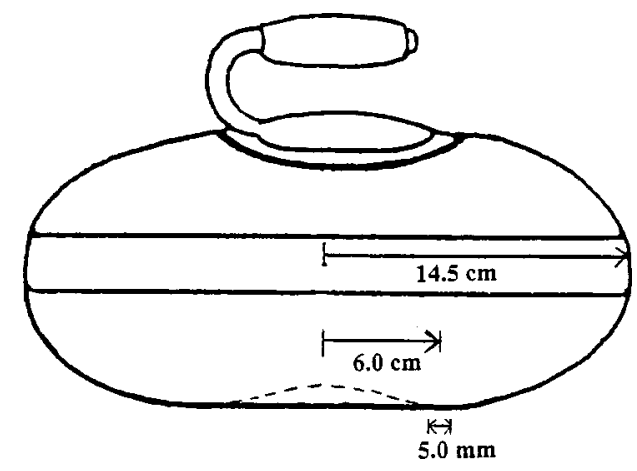

Fig. 7. The profile of a curling rock with a running band radius of $6.0 \mathrm{~cm}$ and width $5.0 \mathrm{~mm}$.

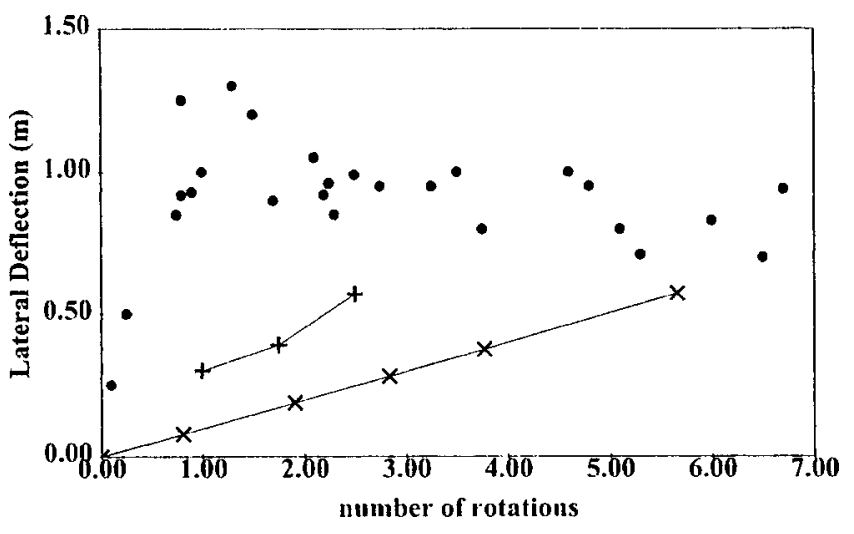

Fig. 8. The dependence of the lateral deflection of a curling rock on the number of completed rotations; $(\boldsymbol{O})$ experimental values, $(\times)$ theoretical values using Eq. (18) with $c_{b} / c_{f}=100,(+)$ theoretical results of Shegelski, Niebergall, and Watton.

will travel further follows from the fact that the component of the frictional force opposing the motion of the rock will decrease with increasing angular velocity. This is similar to the behavior of a rotating hockey puck as described by Voyenli and Eriksen, ${ }^{7}$ and to the behavior of the rotating cylindrical shell as is seen in Fig. 4 where the distance traveled increased with increasing angular velocity.

To determine the actual effect of angular velocity on the amount of curl, data were collected at the local curling rink. Rocks were thrown with various angular velocities along the center line which runs the length of the curling sheet. The lateral deflection of those which stopped within the rings was measured along with the total number of rotations that they completed. The results are shown in Fig. 8. Although the scatter is fairly large, partly due to the experimental technique and partly due to real variations with the rocks, the results show that the amount of curl increases quickly with the total number of rotations, or equivalently, with the initial angular velocity, up to approximately one complete rotation. After this point the total deflection is fairly constant although it does appear that the amount of curl decreases slowly with increasing angular velocity. It was difficult to get results for rotations much less than one for in this case the rock would typically lose its rotation before coming to rest. The results agree with curling practice except that the amount of curl measured did not seem to fall off as quickly with the larger angular velocities as most curlers would believe. Before discussing the various models proposed to explain the curl of a curling rock, the coefficient of kinetic friction between the rock and ice first needs to be determined.

\section{Determination of $\boldsymbol{\mu}_{\boldsymbol{k}}$}

The low values of friction between a sliding object and an ice surface are normally attributed to a thin lubricating water film produced primarily by frictional heating. However, the coefficient of kinetic friction can be determined without consideration of the actual physical mechanism responsible for the retarding force but instead by looking at the problem in terms of energy transfer. ${ }^{8-10}$

Assuming that there are $n$ contact points between the slider and ice, and that these contact areas are square with sides of length $s$, the magnitude of the frictional force per contact point, $f_{c}$, can be expressed as

$$
f_{c}=\mu_{k} F_{n} / n
$$


where $\mu_{k}$ is the coefficient of kinetic friction and $F_{n}$ is the net normal force between the two surfaces. The energy produced at each contact point, $Q_{f}$, by the frictional force during a contact time of $s / v$ is then given by

$$
\begin{aligned}
Q_{f} & =f_{c} v(s / v) \\
& =\mu_{k} F_{n} s / n .
\end{aligned}
$$

The energy produced is used not only for melting the ice at the contact point but will also be conducted into both the ice and into the slider. For sliders with similar thermal properties of a curling rock, it is found ${ }^{8}$ that the bulk of the heat produced at the surface is transferred into the ice. Assuming that the heat produced at each contact point is conducted into a layer of ice of thickness $\delta$ with a temperature differential across the layer of $\Delta T$ equal to the difference between the surface temperature and the bulk ice temperature, we have

$$
Q_{f}=\lambda_{i}\left(s^{2} / \delta\right) \Delta T(s / v),
$$

where $\lambda_{i}$ is the thermal conductivity of the ice. Setting this equal to the heat stored in the layer,

$$
Q_{s}=\left(\rho_{i} s^{2} \delta\right) c_{i} \Delta T / 2,
$$

where $\rho_{i}$ is the density of the ice and $c_{i}$ is the specific heat capacity of the ice, solving for $\delta$, and then substituting back into Eq. (12) results in

$$
Q_{f}=(1 / 2)^{1 / 2} s^{5 / 2}\left(\lambda_{i} \rho_{i} c_{i}\right)^{1 / 2} \Delta T v^{-1 / 2} .
$$

Equating Eqs. (11b) and (14) then leads to the following expression for $\mu_{k}$ :

$$
\mu_{k}=(1 / 2)^{1 / 2} s^{3 / 2} n F_{n}^{-1}\left(\lambda_{i} \rho_{i} c_{i}\right)^{1 / 2} \Delta T v^{-1 / 2} .
$$

Substituting the appropriate values for $\lambda_{i}, \rho_{i}$, and $c_{i}$, equating $s$ to $\left(F_{n} / n P\right)^{1 / 2}$ where $P$ is the interface pressure, and correcting for the fact that the contact points are elliptical and not square, ${ }^{9}$ result in

$$
\mu_{k}=\left(2010 \mathrm{Nm}^{-1} \mathrm{~K}^{-1} \mathrm{~s}^{-1 / 2}\right)\left(n^{1 / 4} P^{-3 / 4}\right) F_{n}^{-1 / 4} \Delta T v^{-1 / 2} .
$$

For an ice temperature of approximately $-5^{\circ} \mathrm{C}$, which is the condition at the local curling rink, and for dwell times, the time it takes the running band to slide over a point on the ice, between $10^{-3}$ and $10^{-1} \mathrm{~s}$, the indentation hardness for ice is approximately $35 \mathrm{MPa}^{11}{ }^{11}$ This is the value that will be used for the interface pressure. In the specific case of the game of curling, the ice is not smooth as it is normally sprayed with water drops at the beginning of the game to give it what is referred to as a pebbled surface. For such a surface the value of $n$ will be taken to be 3, the minimum required for mechanical stability. Using these values along with a value of $5^{\circ} \mathrm{C}$ for $\Delta T$ and a value of $176 \mathrm{~N}$ for $F_{n}$, the weight of a curling rock, results in

$$
\mu_{k}=0.0080 v^{-1 / 2} \text {, }
$$

where $v$ is expressed in $\mathrm{m} / \mathrm{s}$. The $v^{-1 / 2}$ dependence given by Eq. (17) has been experimentally verified ${ }^{8-10}$ for various sliders on ice. To verify whether Eq. (17) also applies to a curling rock, experimental measurements were undertaken at the local curling rink.

A curling rock was manually dragged along the ice and the force required to keep it moving at uniform velocity was measured. It was not possible to obtain usable results for velocities greater than approximately $1 \mathrm{~m} / \mathrm{s}$ with the given

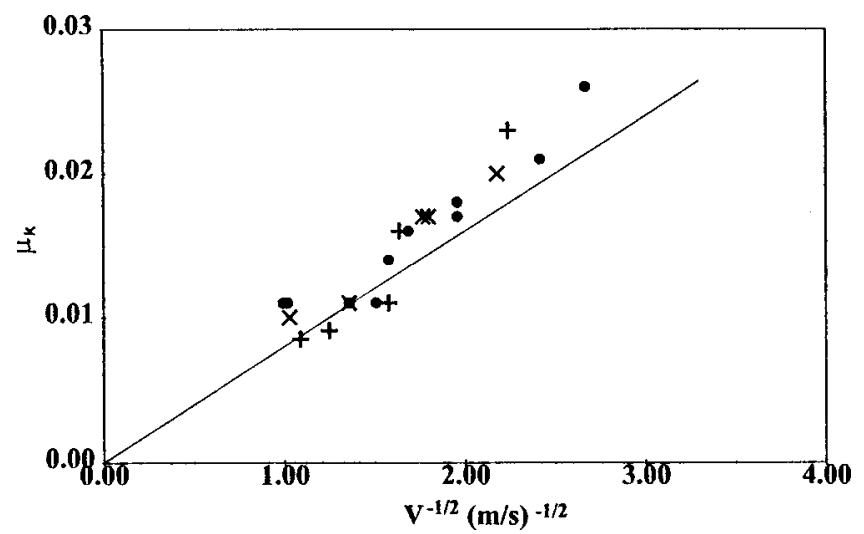

Fig. 9. The dependence of the coefficient of kinetic friction on velocity; ( experimental values for a curling rock, $(\times)$ experimental values for the full curling rock model, $(+)$ experimental values for the half curling rock model, (-) theoretical values as per Eq. (17).

experimental method and for velocities less than $0.15 \mathrm{~m} / \mathrm{s}$ the stick-slip nature of the friction did not allow for any consistent results. The experimental values for $\mu_{k}$ are plotted against $v^{-1 / 2}$ in Fig. 9 along with Eq. (17). As is seen the agreement is quite good considering the approximations used in deriving the theoretical equation and the experimental method. Therefore, it can be concluded that the coefficient of kinetic friction for a curling rock behaves similarly to other sliders running over an ice surface and has the velocity dependence given by Eq. (17).

\section{Front-back asymmetry models}

The frictional force is the only force acting on a curling rock which has components in the plane of the path. Therefore, it is expected that friction should be responsible for the curl of a curling rock. As shown in Fig. 3, the frictional force on the back section of the running band has a component in the correct direction. Two of the curling models use this fact to propose that the curl of a curling rock is due to a difference in the friction between the leading and following halves of the running band. Shegelski, Niebergall, and Watton explain this asymmetry as being due to different amounts of wet and dry friction on the two halves. The wet friction is modeled as being due to hydrodynamic drag, $k v^{2}$, while the dry friction is taken to be constant. This is in disagreement with the experimental data presented in Fig. 9 and previous results for sliders on ice. Johnston argues that if the frictional force decreased with increasing normal force, the friction at the back half of the running band will be greater than at the front and the rock will have the correct curl. Again, this is in disagreement with experimental results ${ }^{8,10}$ for sliders on ice. Although $\mu_{k}$ decreases with the normal force, as per Eq. (16), the frictional force, $\mu_{k} F_{n}$, increases with $F_{n}$.

Although these specific models would appear to be inconsistent with experimental results and theoretical models of friction on ice, the idea that a front-back asymmetry of the frictional force would lead to the correct lateral deflection is correct. If the frictional force were greater at the back half due to any conditioning of the ice from the front half passing over it first, the curling rock would curl in the correct direction. To determine how large an effect would be required, the path of a curling rock was computed using Eqs. (7)-(9). The coefficient of kinetic friction was set as 


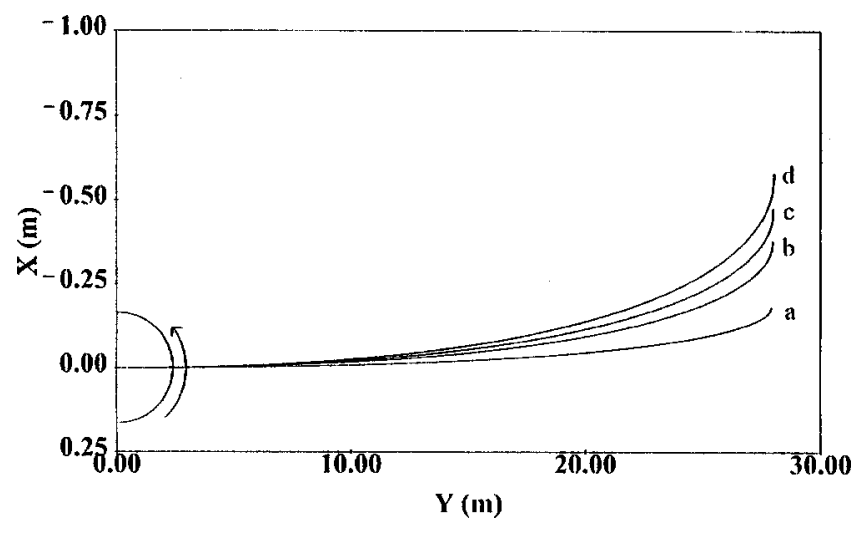

Fig. 10. The theoretical paths of curling rocks with $v_{y 0}=2 \mathrm{~m} / \mathrm{s}, \quad \omega_{0}$ $=1.5 \mathrm{rad} / \mathrm{s}$, and $c_{b} / c_{f}=2: 1$ (a), 5:1 (b), 10:1 (c), and 100:1 (d).

$$
\begin{aligned}
\mu_{k} & =c_{f} v^{-1 / 2} \quad \text { for } 0 \leqslant \theta<\pi \\
& =c_{b} v^{-1 / 2} \text { for } \pi \leqslant \theta<2 \pi,
\end{aligned}
$$

and to conform to Eq. (17) $\left(c_{f}+c_{b}\right) / 2$ was set to 0.0080 . The initial translational velocity of the rock was set at $2.0 \mathrm{~m} / \mathrm{s}$ and the initial angular velocity was set at $1.5 \mathrm{rad} / \mathrm{s}$, which resulted in the rock stopping after traveling $28 \mathrm{~m}$. Figure 10 shows the resulting paths for various ratios of $c_{b} / c_{f}$. In order to get the rock to curl even $0.5 \mathrm{~m}$, at the given initial angular velocity, required that the friction on the back half of the running band be on the order of 10-100 times greater than the friction at the front half. Only for much larger angular velocities than is found in the game will the rock curl up to $1 \mathrm{~m}$. This result is independent of the form of the asymmetry and the previous mentioned curling models require similar large differences. For example, in Shegelski, Niebergall, and Watton's model for curling rock velocities less than $0.25 \mathrm{~m} / \mathrm{s}$, the ratio of the friction at the back half to that of the front is greater than 20:1.

In order to determine if such a large front-back asymmetry exists with the frictional force, models of a full and a half curling rock were constructed. The profile of the bottom of a curling rock was cut into two acrylic disks. Mass was then added to one of the disks so as to model an 18-kg curling rock. The other disk was cut in half and mass was added up to a total $9 \mathrm{~kg}$ so as to model the front half of a curling rock. The two models were then dragged along the ice at various velocities and the resulting coefficients of kinetic friction were determined. There was no significant difference found between the coefficients of kinetic friction for the full model and the half model and both agreed well with the results for the real curling rock, as can be seen in Fig. 9. If the friction at the back half was much greater than at the front half the coefficient of friction would have been much greater for the full model than the half model. Although the experimental setup does not fully model the motion of a slowly rotating curling rock traveling down a sheet of ice, it would be difficult to explain how one could get an extreme asymmetry in the frictional force in one case and not in the other.

The front-back asymmetry model also breaks down when one considers the effect of angular velocity on the curl. The larger the initial angular velocity, the larger the lateral components of friction around the band will be and the greater the expected curl. Figure 8 includes the dependence of the lateral deflection of a curling rock on the amount of rotation for the front-back asymmetry model given by Eq. (18) with

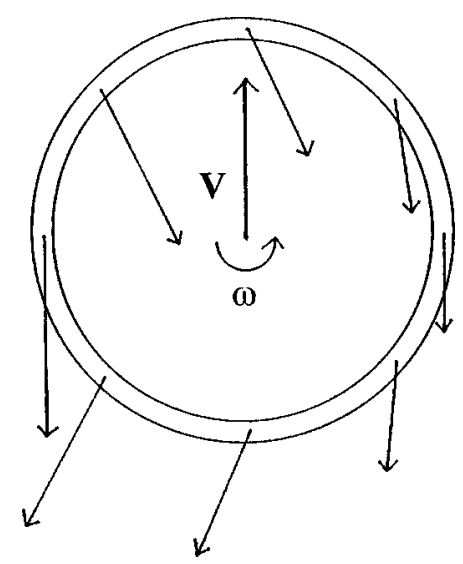

Fig. 11. The resulting frictional force at various points around the running band for a curling rock where $\mu_{k}$ decreases with increasing velocity.

$c_{b} / c_{f}$ equal to 100 along with the results of Shegelski, Niebergall, and Watton. As is seen the curl for these models is approximately proportional to the total amount of rotation and therefore to the initial angular velocity, in disagreement with the experimental data and standard curling practice. Thus a front-back asymmetry in the frictional force would not appear to be the reason behind the curl of a curling rock.

\section{E. Left-right asymmetry models}

As shown in Fig. 1(b), the rotation of a cylindrical shell or a curling rock will cause the magnitude of the velocity at different positions around the running band to vary. In the case of a curling rock this will in turn result in the magnitude of the frictional force, as per Eq. (17), varying around the running band. Figure 11 shows the result with the frictional force on the left side being greater than the frictional force on the right side. Figure 12 shows how the coefficient of kinetic friction will vary on the two sides of the running band over the transit of a curling rock with an initial translational velocity of $2.0 \mathrm{~m} / \mathrm{s}$ and an initial angular velocity of 1.5 $\mathrm{rad} / \mathrm{s}$. The difference varies from approximately $5 \%$ at the beginning to over $30 \%$ at the end of the run. Denny proposed a left-right asymmetry in the frictional force but modeled the coefficient of kinetic friction as being velocity independent. This model is in conflict with the previously mentioned experimental results for sliders and curling rocks on ice.

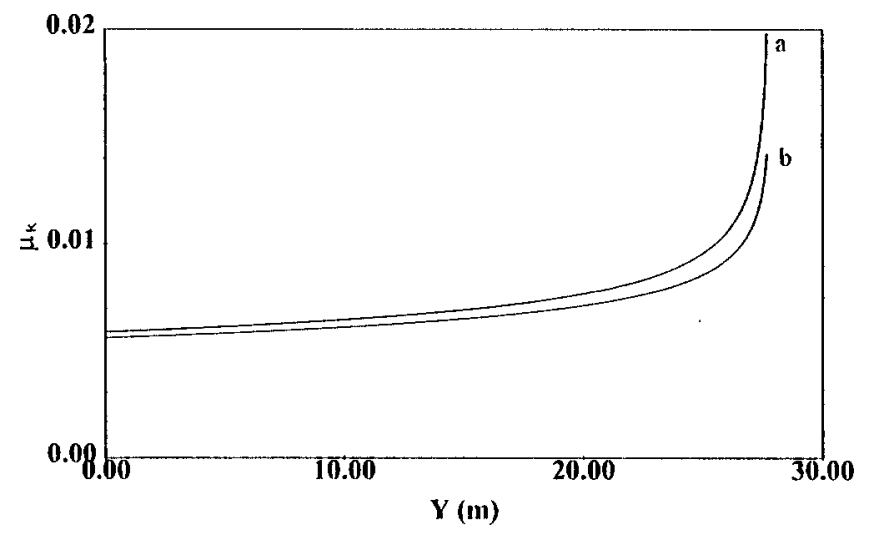

Fig. 12. The variation in $\mu_{k}$ on the (a) far left and (b) far right of the running band of a curling rock which is rotating counterclockwise as in Fig. 11. 
Unfortunately, the left-right frictional asymmetry model by itself does not explain why a curling rock curls. As can be seen in Fig. 11 there is no net lateral force. Denny overcomes this problem by proposing that the torque acting on the rock rotates the reference frame of the rock relative to the ice and that this causes the velocity of the center of mass to change direction. However, this explanation is in direct conflict with Newton's second law as it argues that the rock can deflect laterally without any net lateral force acting.

\section{F. Observations and hypothesis}

As stated previously, it is expected that the frictional force between the curling rock and the ice is responsible for the curl of a rock. Unless a rotating curling rock causes the normal force to deviate from the vertical direction, there does not seem to be any other option. The importance of friction in causing the curl of a rock is highlighted by several key features of the game. First, it is normal practice for several members of the rock throwing team to vigorously sweep the ice ahead of the rock. This is done in order to cause a rock to travel further by reducing the amount of friction or at other times it is done to decrease the amount a rock curls in order to avoid collisions. Second, the running band is purposely left unpolished, unlike the rest of the polished rock, and therefore has a slightly roughened surface. The rougher the band, or the sharper the stone in curling parlance, the more the rock curls. Third, as previously mentioned, the ice is sprayed with water drops prior to a game to give the ice sheet a pebbled surface in order to reduce the frictional force and make it easier to throw the rocks the length of the ice. However, the pebble also affects the amount of curl and often rocks do not curl as much at the beginning of a game when the pebble is fresh. In addition, on ice that has not been pebbled the rocks will not curl as much. All these observations certainly seem to indicate that friction is responsible for the curl of a rock.

However, it is also observed that the curl of a rock is not always smooth as it moves down the ice. Some thrown rocks deflect relatively sharply from their path as they seem to " catch" something on the ice. This deviation from their path is normally in the same direction that the rock is curling. In addition, rocks will often pivot about their slow side, the left side in Fig. 1(b), just prior to coming to rest. These additional observations, combined with the severe stick-slip nature of ice that was observed during the measurements of the coefficient of kinetic friction, indicate that the frictional force between the curling rock and the ice is partly adhesive in nature. If this is the case, a frictional model based on Eq. (2a) is inadequate. The adhesive nature of the force would be expected to result in a pivoting-like action about the contact point in addition to the retardation expressed by Eqs. (2a) and (17). Contact points on the left or slow side of the running band would cause the rock to curl counterclockwise, with respect to Fig. 1(b), while contact points on the right side would cause the rock to curl clockwise.

The magnitude of the effect of the pivoting action on the deflection of the curling rock would be expected to be proportional to the angular momentum about the given contact point. This follows from the fact that the angular momentum about the pivot point will be identical before and after any arresting action. The magnitude of the angular momentum of the curling rock about a contact point on the left or slow side of the running band is greater than an equivalent point on the right side. As an example, the angular momentum about con- tact points on the far left, $L_{L}$, and far right sides, $L_{R}$, of a running band of radius $R$ for a curling rock traveling down the ice with a velocity $v$, and angular velocity $\omega$, is given by

$$
\begin{aligned}
& \left|L_{L}\right|=m v R+I \omega, \\
& \left|L_{R}\right|=m v R-I \omega,
\end{aligned}
$$

where $m$ is the mass and $I$ is the moment of inertia of the rock about a vertical axis through its center. This left-right asymmetry with regards to the angular momentum would result in a preferential pivoting about the left side, leading to a net deflection or curl of the rock in that direction, which is what is observed. In addition the left-right asymmetry in the frictional force, shown in Fig. 12, would also be expected to play a part. The greater frictional force on the left side of the running band would indicate a greater adhesive effect and therefore a greater pivoting action. This would again result in preferential pivoting about the slow side and a net deflection in that direction. Unfortunately, attempts to model the preferential pivoting inevitably lead to a curl which increases with the angular velocity of the rock, in disagreement with the experimental results. Obviously, a deeper understanding of the adhesive nature of the frictional force and the resulting pivoting action is required in order to correctly model the resulting deflection of the curling rock and its dependence on the parameters of the problem.

\section{CONCLUSION}

The lateral deflection of a rotating cylindrical shell sliding on end over a dry horizontal table was shown to be due to the increased normal force at the front half of the running band. Experimentally measured paths of such cylinders were found to match well with the theoretically derived paths. The amount of lateral deflection increases with both the angular velocity and the height of the cylinder.

The motion of a curling rock is more difficult to explain. Front-back frictional asymmetry models were found to disagree with theoretical models of friction on ice and with experimental measurements using half and full models of curling rocks. They also predict that the lateral deflection should increase with the total number of rotations or, equivalently, the initial angular velocity of the curling rock, which is in conflict with experimental results. Left-right frictional asymmetry models based on the conventional frictional model of Eq. (2a) do not result in any net lateral force and therefore by themselves cannot explain the curl of a curling rock. Observations of the motion of curling rocks indicate that the frictional force is partly adhesive in nature. It is hypothesized that this will result in preferential pivoting about the slow side of the rock and that this is the primary mechanism behind the curl of a curling rock. Unfortunately, a model based on this hypothesis has not yet been found which agrees with the observed dependence of a curling rock's deflection on angular velocity.

\section{ACKNOWLEDGMENTS}

I would like to thank Dr. W. Weller for discussions and assistance in constructing the curling rock models and G. Twanow and N. Penner for help in carrying out the tests at the Nanaimo curling rink. 
${ }^{1}$ J. M. Daniels, "Comment on 'On the motion of an ice hockey puck,", Am. J. Phys. 54 (9), 777-778 (1986).

${ }^{2}$ G. W. Johnston, "The dynamics of a curling stone," Can. Aeronaut. Space J. 27, 144-160 (1981).

${ }^{3}$ M. R. A. Shegelski, R. Niebergall, and M. A. Watton, "The motion of a curling rock," Can. J. Phys. 74, 663-670 (1996).

${ }^{4}$ M. Denny, "Curling rock dynamics," Can. J. Phys. 76, 295-304 (1998)

${ }^{5}$ E. Lukowich, The Curling Book (Western Producer Prairie Books, Saskatoon, 1981).
${ }^{6}$ M. Mulvoy and E. Richardson, Curling Techniques and Strategy (Lippincott, New York, 1973).

${ }^{7} \mathrm{~K}$. Voyenli and E. Eriksen, "On the motion of an ice hockey puck," Am. J. Phys. 53 (12), 1149-1153 (1985)

${ }^{8}$ D. Evans, "Kinetic friction of ice," Proc. R. Soc. London, Ser. A 347, 493-512 (1976).

${ }^{9}$ P. Oksanem, "Mechanism of friction of ice," Wear 78, 315-324 (1982).

${ }^{10} \mathrm{M}$. Akkok, "Parameters affecting the kinetic friction of ice," J. Tribol. 109, 552-560 (1987).

${ }^{11}$ P. V. Hobbs, Ice Physics (Oxford U.P., London, 1974). 